\title{
Optimisation exergo-économique d'une turbine à gaz
}

\author{
A. Dobrovicescu' et L. Grosu² \\ 1 Université Politehnica de Bucarest, Splaiul Independentei, 313, Sect. 6, Bucureflti - Roumanie \\ 2 Université Paris Ouest Nanterre La Défense, Pôle Scientifique et Technologique de Ville d'Avray, Laboratoire Énergétique, \\ Mécanique et Électromagnétisme, 50 rue de Sèvres, 92410 Ville d'Avray - France \\ e-mail: adobrovicescu@yahoo.com - lavinia-grosu@u-paris10.fr
}

Résumé - Optimisation exergo-économique d'une turbine à gaz - Ce travail concerne l'optimisation exergo-économique d'une turbine à gaz. La méthode d'optimisation exergo-économique est comparée avec la méthode thermoéconomique. Il est mis en évidence que l'optimisation exergoéconomique offre des études et raisonnements supplémentaires qui ne sont pas disponibles en se limitant à une optimisation énergétique classique. L'objectif d'une optimisation exergo-économique d'un système énergétique est de trouver la structure optimale et les paramètres de fonctionnement optimum qui minimisent le coût global du système, sous des contraintes imposées de fonctionnement, de maintenance et d'impact sur l'environnement. Cette optimisation est réalisée en étudiant l'évolution de paramètres exergétiques locaux et globaux.

\begin{abstract}
Exergoeconomic Optimization of a Gas Turbine - The paper deals with the exergoeconomic optimization of a gas turbine system. A parallel between thermoeconomic and exergoeconomic optimization is carried on. It is pointed out that exergoeconomics offers additional capabilities in optimization that are not available with the conventional technic optimization. The goal of exergoeconomic design optimization of an energy systems is to find the structure and the values of the system parameters that minimize the cost of final products considering restrictions imposed by the desired reliability, maintainability, operability and environmental impact of the system, using local or global exergetic parameters.
\end{abstract}




\section{NOMENCLATURE}

$\dot{C} \quad$ Coût par unité de temps $\left(€ . \mathrm{s}^{-1}\right)$

c Coût unitaire $\left(€ . \mathrm{kJ}^{-1}\right)$

Cc Chambre de combustion

$C A$ Coût d'acquisition (€)

$\dot{C} I \quad$ Coût de l'irréversibilité interne par unité de temps $\left(€ . \mathrm{s}^{-1}\right)$

$C p$ Compresseur

$\dot{E x} \quad$ Flux d'exergie $(\mathrm{kW})$

f $\quad \dot{Z}_{k} /\left(\dot{Z}_{k}+\dot{C}_{k}\right)$ facteur exergo-économique (-)

$G$ Générateur électrique

$\bar{g}^{0} \quad$ Fonction molaire de formation $\left(\mathrm{kJ}^{\mathrm{k}} \mathrm{kmol}{ }^{-1}\right)$

$\bar{h} \quad$ Enthalpie molaire $(\mathrm{kJ} / \mathrm{kmol})$

$\bar{h}^{0} \quad$ Enthalpie molaire de formation $\left(\mathrm{kJ}^{\mathrm{k}} \mathrm{kmol}^{-1}\right)$

$\dot{H} \quad$ Flux d'enthalpie $(\mathrm{kW})$

I Flux de destruction d'exergie due aux irréversibilités internes $(\mathrm{kW})$

$\dot{L} \quad$ Puissance mécanique $(\mathrm{kW})$

$\dot{m} \quad$ Débit massique $\left(\mathrm{kg} . \mathrm{s}^{-1}\right)$

$n \quad$ Nombre de $\mathrm{kmol}(\mathrm{kmol})$

$\hat{n} \quad$ Nombre de kmol par kmol de combustible $\left(\mathrm{kmol} \mathrm{kmol}_{\mathrm{Cb}}{ }^{-1}\right)$

$\mathrm{Pa}$ Préchauffeur d'air

$P C I$ Pouvoir calorifique inférieur $\left(\mathrm{kJ}^{\mathrm{kmol}} \mathrm{Cb}^{-1}\right)$

$P e \quad$ Puissance effective $(\mathrm{kW})$

$r_{k} \quad\left(c_{P, k}-c_{C b, k}\right) / c_{C b, k}$ augmentation relative du coût (-)

$\bar{s} \quad$ Entropie molaire $\left(\mathrm{kJ} \cdot \mathrm{kmol}^{-1} \cdot \mathrm{K}^{-1}\right)$

$\bar{s}^{0} \quad$ Entropie molaire absolue $\left(\mathrm{kJ} \cdot \mathrm{kmol}^{-1} \cdot \mathrm{K}^{-1}\right)$

$t$ Turbine

$T$ Température (K)

$\dot{Q} \quad$ Flux de chaleur $(\mathrm{kW})$

$\dot{Z} \quad$ Coût d'amortissement par unité de temps $\left(€ . \mathrm{s}^{-1}\right)$

$x \quad$ Taux molaire (-)

y $\quad \dot{I} / \dot{E} x_{C b}^{T O T}$ rapport du flux de destruction d'exergie par le flux d'exergie dépensée (combustible du système) (-)

\section{Lettres grecques}

$\eta \quad$ Rendement (-)

$\pi \quad$ Rapport de compression (-)

$\varepsilon \quad$ Perte relative de pression (-)

\section{Indices}

a Air

Cc Chambre de combustion

$\mathrm{Cb}$ Combustible

Cp Compresseur

$\begin{array}{ll}e & \text { Échappement } \\ e x & \text { Exergétique } \\ s & \text { Isentropique } \\ g & \text { Gaz } \\ k & \text { Composant, zone } \\ \text { max } & \text { Maximum } \\ P & \text { Produit } \\ P a & \text { Préchauffeur d'air } \\ r e f & \text { Référence } \\ t & \text { Turbine } \\ \text { tot } & \text { Total }\end{array}$

\section{Exposants}

\author{
$0 \quad$ Équilibre \\ pe Perte \\ TM Thermomécanique \\ TOT Total
}

\section{INTRODUCTION}

L'optimisation des systèmes énergétiques complexes devient extrêmement lourde due au nombre important d'équations, de contraintes et de variables à prendre en compte dans les modèles mathématiques. Un point faible des méthodes analytiques et numériques d'optimisation énergétique est l'impossibilité d'indiquer avec précision l'influence de la structure d'un système sur le potentiel d'amélioration de son efficacité et l'impact sur le coût global [1,2].

L'exergo-économie offre des possibilités d'optimisation supplémentaires par rapport aux méthodes d'optimisation conventionnelles. Le but de l'optimisation exergo-économique est de définir la structure et les paramètres de fonctionnement qui minimisent le coût des produits finaux, tenant compte des contraintes imposées par rapport aux conditions de maintenance et de fonctionnement du système et par rapport à son impact sur l'environnement.

L'intérêt des corrélations thermoéconomiques et exergoéconomiques a été prouvé dans des travaux antérieurs. Dans la littérature, on trouve des corrélations définissant le coût d'investissement de différents composants en fonction de paramètres physiques [3-6].

Afin d'illustrer le potentiel intéressant d'analyse et d'optimisation de l'approche exergétique, nous avons choisi d'étudier une turbine à gaz simple et avec récupération.

\section{MODĖLE THERMOÉCONOMIQUE DE LA TURBINE À GAZ SIMPLE}

Le schéma du système et le cycle de fonctionnement associé en diagramme $T-s$, sont présentés dans la figure 1. 


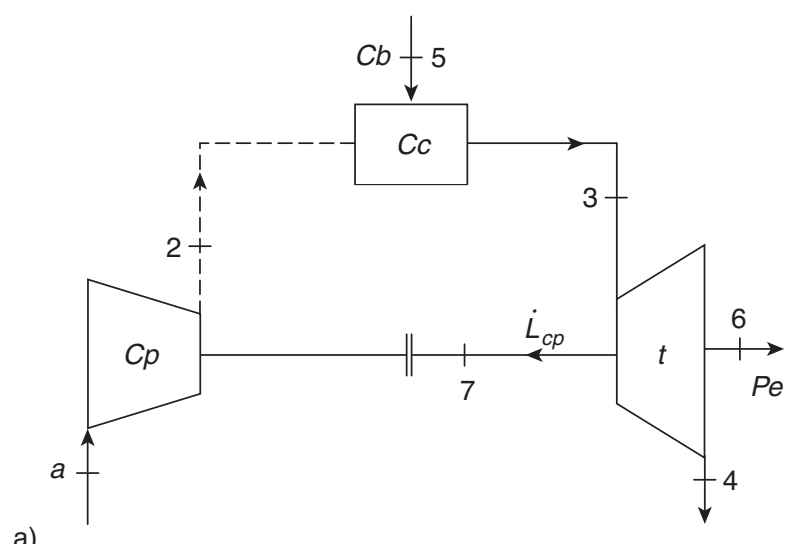

a)

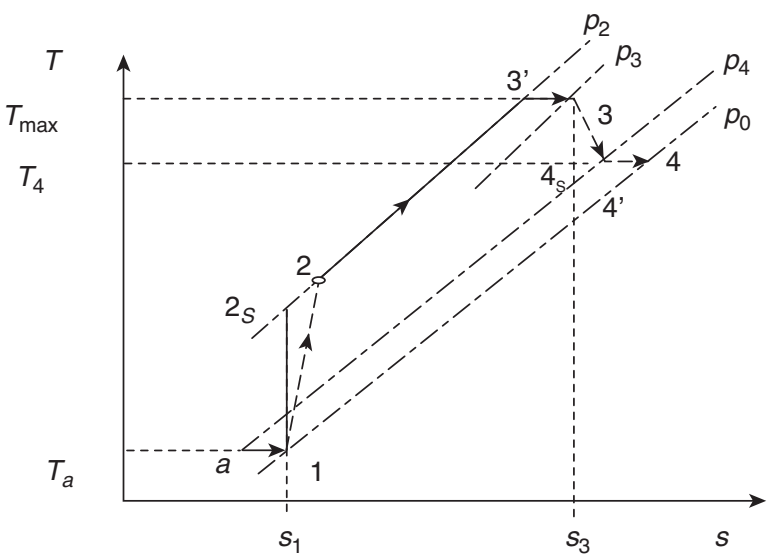

b)

Figure 1

a) Schéma de la turbine à gaz simple.

b) Diagramme $T$-s : cycle de fonctionnement de la turbine à gaz simple.

Les paramètres du problème d'optimisation sont les suivants :

$$
\begin{gathered}
P e=65 \mathrm{MW} \\
\eta_{C c}=0,98 \\
\varepsilon_{a}=\Delta p_{a-1} / p_{a}=0,05 \\
\varepsilon_{e}=\Delta p_{4^{\prime}-4} / p_{4}=0,05 \\
\varepsilon_{C c}=\Delta p_{3^{\prime}-3} / p_{2}=0,05
\end{gathered}
$$

Le carburant utilisé est du $\mathrm{CH}_{4}$, le comburant est de l'air avec la composition molaire suivante :

$$
\begin{aligned}
& x_{\mathrm{N}_{2}}^{0}=0,7748 ; x_{\mathrm{O}_{2}}^{0}=0,2059 ; \\
& x_{\mathrm{CO}_{2}}^{0}=0,0003 ; x_{\mathrm{N}_{2}}^{0}=0,019 .
\end{aligned}
$$

\subsection{Calcul des paramètres d'état caractéristiques du système}

La composition du gaz de travail étant modifiée par la réaction chimique dans la chambre de combustion, les paramètres d'état sont déterminés par rapport à un état de référence commun à tous les constituants : $\bar{g}^{0}, \bar{h}^{0}, \bar{s}^{0}, T_{r e f}=298,15 \mathrm{~K}$ et $p_{\text {ref }}=1$ bar.

Par conséquent :

$$
\bar{h}(T)=\bar{h}^{0}(T)
$$

et

$$
\bar{s}(T, p)=\bar{s}^{0}(T)-\bar{R} \cdot \ln \frac{p}{p_{\text {ref }}}
$$

Le gaz de travail est supposé un gaz parfait. L'enthalpie et l'entropie du mélange de gaz sont exprimées comme suit :

$$
\begin{gathered}
\bar{h}(T)=\sum x_{k} \cdot \bar{h}_{k}(T) \\
\bar{s}(T, p)=\sum x_{k} \cdot \bar{s}_{k}\left(T, p_{k}\right)
\end{gathered}
$$

avec $p_{k}=x_{k} \cdot p$, la pression partielle du composant $k \mathrm{du}$ mélange (à pression $p$ ).

\subsection{Calcul de la composition de produits de composition}

Si on néglige la variation des énergies potentielles et cinétiques dans la chambre de combustion, on peut écrire le bilan énergétique de ce composant sous la forme simple suivante :

$$
\dot{Q}_{C c}^{p e}=\Delta \dot{H}
$$

où, $\dot{Q}_{C c}^{p e}$ représente le flux de chaleur perdu.

Pour $1 \mathrm{kmol}$ de combustible, le bilan énergétique de la chambre de combustion devient [7] :

$$
\left(\eta_{\text {comb }}-1\right) P C I=\hat{n}_{g} \cdot \overline{h_{g}}\left(T_{3}\right)-\overline{h_{C b}}\left(T_{5}\right)-\hat{n}_{a} \cdot \overline{h_{a}}\left(T_{2}\right)
$$

où :

$$
\begin{gathered}
\hat{n}_{g} \cdot \bar{h}_{g}\left(T_{3}\right)=\hat{n}_{\mathrm{O}_{2}} \cdot \bar{h}_{\mathrm{O}_{2}}\left(T_{3}\right)+\hat{n}_{\mathrm{CO}_{2}} \cdot \bar{h}_{\mathrm{CO}_{2}}\left(T_{3}\right)+ \\
\hat{n}_{\mathrm{N}_{2}} \cdot \bar{h}_{\mathrm{N}_{2}}\left(T_{3}\right)+\hat{n}_{\mathrm{H}_{2} \mathrm{O}} \cdot \bar{h}_{\mathrm{H}_{2} \mathrm{O}}\left(T_{3}\right)
\end{gathered}
$$

Afin de trouver une géométrie et un fonctionnement optimum du système, on a identifié les variables de décision suivantes :

- le rapport de compression $\pi=p_{2} / p_{1}$,

- la température des gaz brûlés $T_{3}$,

- le rendement isentropique du compresseur $\eta_{s C p}$,

- le rendement isentropique de la turbine $\eta_{s t}$.

La fonction objective à minimiser représente le coût total du produit, qui est la somme du coût du combustible (dépense) et du coût d'investissement du système. Le coût d'investissement prend en compte les coûts d'acquisition, le coût de maintenance du système et les différentes taxes et coûts d'assurances. 


$$
\dot{C}=\dot{C}_{C b}+\sum \dot{Z}_{k}
$$

où $\dot{Z}_{k}\left(€ . \mathrm{s}^{-1}\right)$ représente le coût d'amortissement du prix de l'équipement $k$.

Les coûts d'acquisition des équipements sont exprimés par les corrélations économiques suivantes [3-5], en fonction des variables de décisions correspondantes :

$$
\begin{gathered}
C A_{C p}=\frac{65,058 \cdot \dot{m}_{a}}{0,9-\eta_{s C p}} \cdot \frac{p_{2}}{p_{1}} \cdot \ln \frac{p_{2}}{p_{1}} \\
C A_{C c}=\frac{42,168 \cdot \dot{m}_{a}}{0,995-\frac{p_{3}}{p_{2}}}\left(1+\exp \left(0,018 \cdot T_{3}-26,4\right)\right. \\
C A_{t}=\frac{438,62 \cdot \dot{m}_{g}}{0,92-\eta_{s t}} \ln \frac{p_{3}}{p_{44}} \cdot\left(1+\exp \left(0,036 \cdot T_{3}-54,4\right)\right)
\end{gathered}
$$

On considère que le système fonctionne 8000 heures par an.

\section{OPTIMISATION THERMOÉCONOMIQUE DE LA TURBINE À GAZ}

Le modèle thermoéconomique repose sur le premier principe de la thermodynamique appliqué aux différents composants du système et sur l'ensemble de corrélations économiques.

La fonction objective (8) a été soumise à une procédure de minimisation dans des conditions restrictives imposées par les équations du bilan énergétique.

La recherche d'une solution optimale a été réalisée par les méthodes "Direct search method" et "Variable metric method" $[1,2]$.

Les valeurs optimales des variables de décision obtenues avec la première méthode sont :

$$
p_{2} / p_{1}=11,67 ; T_{3}=1460 \mathrm{~K} ; \eta_{s C p}=0,815 ; \eta_{s t}=0,874 ;
$$

pour lesquelles on obtient un coût minimum du produit égal à $\dot{C}=2,446 €$ s. $^{-1}$.

Avec la méthode "Variable metric method" les valeurs des variables de décision sont :

$$
p_{2} / p_{1}=10 ; T_{3}=1200 \mathrm{~K} ; \eta_{s C p}=0,828 ; \eta_{s t}=0,876 ;
$$

avec un coût minimum correspondant de $\dot{C}=2,98 € . \mathrm{s}^{-1}$.

La différence entre les valeurs trouvées par les deux méthodes nous conduit à penser que le minimum trouvé par la deuxième méthode n'est qu'un minimum local.

Peu importe la performance de la méthode mathématique de recherche d'optimum, on perd vite le contact avec le modèle physique et la procédure d'optimisation n'offre aucune indication d'amélioration à faire au niveau du système.
Seule une étude approfondie interne du système et la compréhension du mécanisme de dépense interne des ressources et des coûts additionnels correspondants (par dépense locale de combustible) offrent une stratégie transparente de recherche de solution optimale.

Cette analyse plus fine du système peut être réalisée en utilisant le concept d'exergie et les corrélations économiques. Elle porte le nom d'analyse exergo-économique [8-19].

\section{OPTIMISATION EXERGO-ÉCONOMIQUE DE LA TURBINE À GAZ}

Le modèle mathématique basé sur le premier principe de la thermodynamique a été complété avec les bilans exergétiques qui ont permis le calcul des destructions et des pertes d'exergie au niveau de chaque sous-système [7].

Trois zones fonctionnelles ont été mises en évidence :

- le compresseur,

- la chambre de combustion,

- la turbine.

Des courants d'énergie et de matière et des coûts unitaires associés sont établis à l'intérieur du système. Le système d'équations qui permet le calcul des coûts de chaque courant d'énergie ou de matière est présenté ci-dessous [20-23] :

$$
\begin{gathered}
\dot{C}_{a}+\dot{C}_{7}-\dot{C}_{2}+\dot{Z}_{C p}=0 \\
\dot{C}_{2}+\dot{C}_{5}-\dot{C}_{3}+\dot{Z}_{C c}=0 \\
\dot{C}_{3}-\dot{C}_{7}-\dot{C}_{6}+\dot{Z}_{t}=0 \\
\dot{C}_{7} \cdot P e-\dot{C}_{6} \cdot \dot{L}_{C p}=0 \\
\dot{C}_{a}=c_{a} \cdot \dot{E} x_{a}^{T M} \\
\dot{C}_{5}=c_{5} \cdot \dot{E} x_{5}^{T O T}
\end{gathered}
$$

Afin d'estimer le coût de la destruction d'exergie de chaque zone, on définit localement un produit et un combustible (ressource).

La destruction locale d'exergie $\dot{I}_{k}$ due aux irréversibilités internes représente la part du combustible local qui est consommée dans le processus et son coût unitaire est celui du combustible local.

Ainsi, le coût de la destruction locale due aux irréversibilités internes est le produit du coût unitaire du combustible local par le flux de destruction d'exergie :

$$
\dot{C} I_{k}=c_{C b, k} \cdot \dot{I}_{k}[€ / \mathrm{s}]
$$

Dans l'expression (18), le coût unitaire du combustible local prend en compte les coûts d'investissement, de main d'œuvre, des taxes et assurances (12-17).

La recherche de l'optimum est orientée par l'évolution des coûts de destruction d'exergie par zone, par le coût de revient de l'amortissement des équipements, par les rendements 


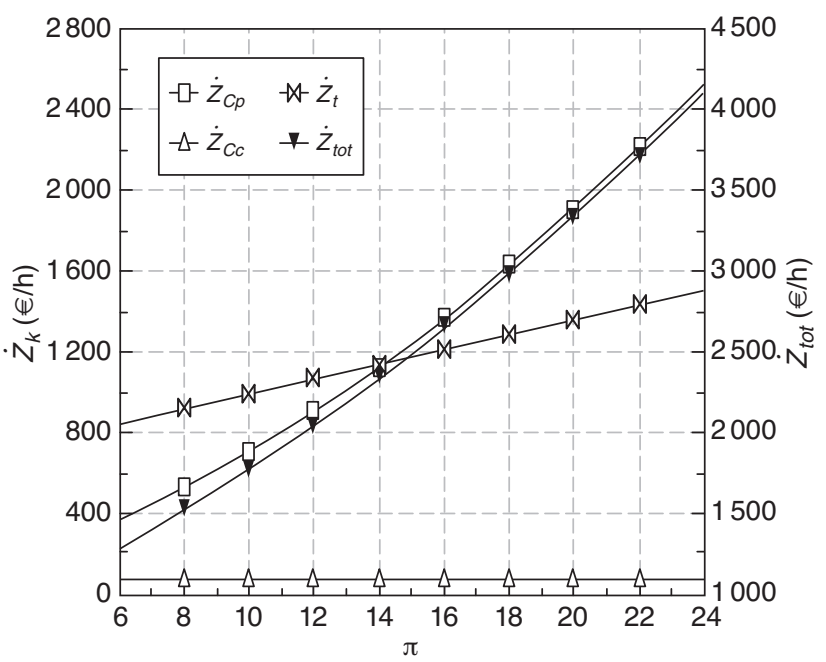

Figure 2

Influence du rapport de compression sur le coût d'amortissement du capital $\left(T_{3}=1460 \mathrm{~K} ; \eta_{s C_{p}}=0,815\right.$; $\left.\eta_{s t}=0,874\right)$.

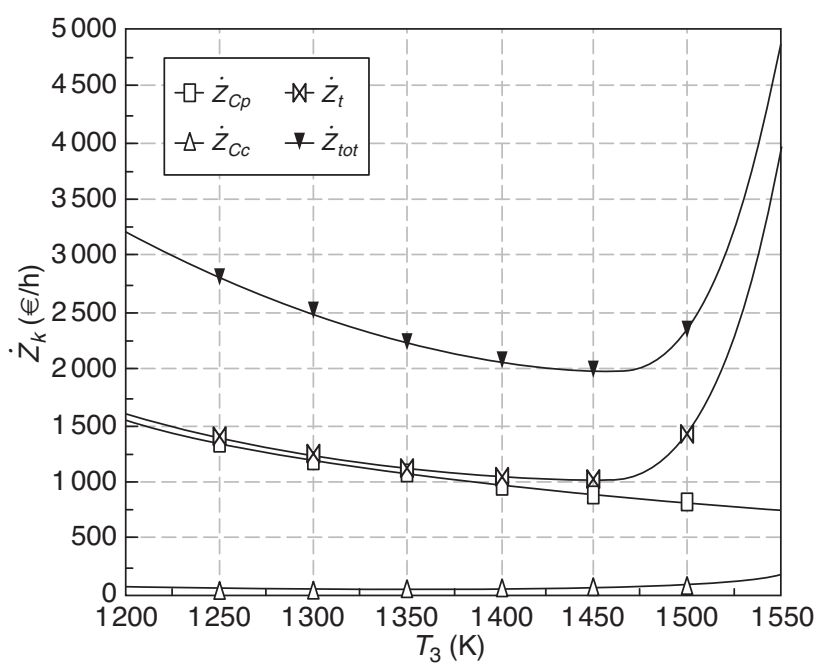

Figure 4

Influence de la température $T_{3}$ à l'entrée dans la turbine sur le coût d'amortissement du capital $\left(p_{2} / p_{1}=11,67\right.$; $\left.\eta_{s C p}=0,815 ; \eta_{s t}=0,874\right)$.

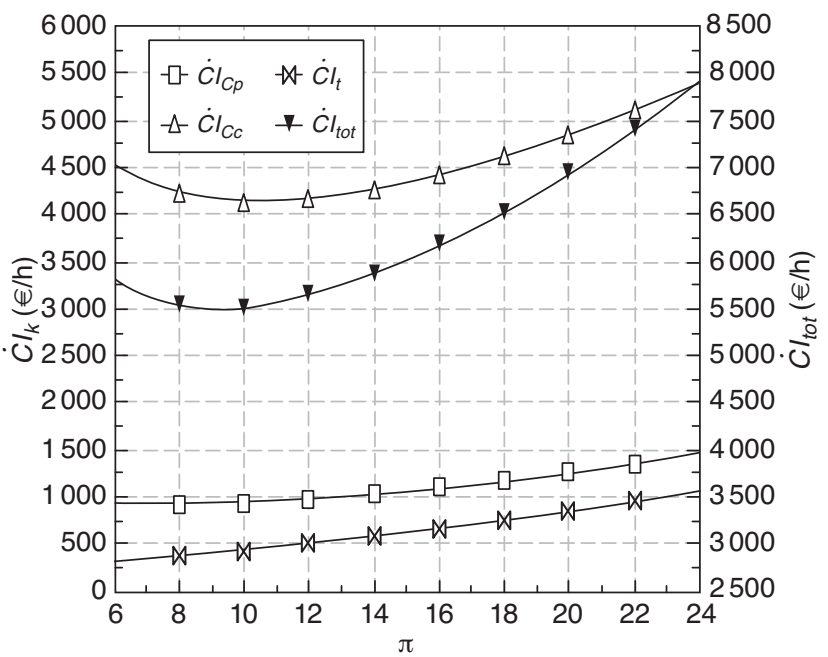

Figure 3

Influence du rapport de compression sur le coût de la destruction d'exergie $\left(T_{3}=1460 \mathrm{~K} ; \eta_{s C p}=0,815\right.$; $\left.\eta_{s t}=0,874\right)$.

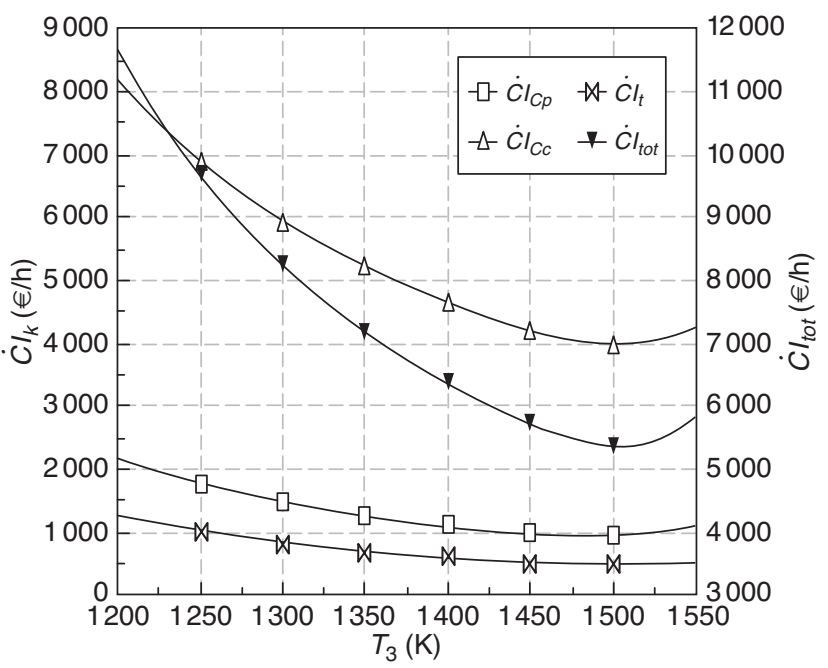

Figure 5

Influence de la température $T_{3}$ à l'entrée de la turbine sur le coût de la destruction d'exergie $\left(p_{2} / p_{1}=11,67\right.$; $\left.\eta_{s C p}=0,815 ; \eta_{s t}=0,874\right)$. exergétiques de chaque zone et par les coefficients exergoéconomiques [24].

La dynamique de la variation des coûts de destruction d'exergie et du coût de revient de l'investissement est prise en considération afin de modifier les valeurs de certaines variables de décision.

Les résultats des études de sensibilité de différents paramètres sur le coût de la destruction d'exergie et sur le coût d'amortissement du capital sont présentés dans les figures 2-9.
Cette étude paramétrique permet de remarquer :

- une élévation rapide du coût d'amortissement du compresseur avec l'augmentation du rapport de compression $\pi$ et l'élévation du rendement isentropique du compresseur $\eta_{s C p}$ (fig. 2 et 6);

- un minimum du coût de la destruction d'exergie dans la chambre de combustion pour un taux de compression $\pi=10$ (fig. 3); 


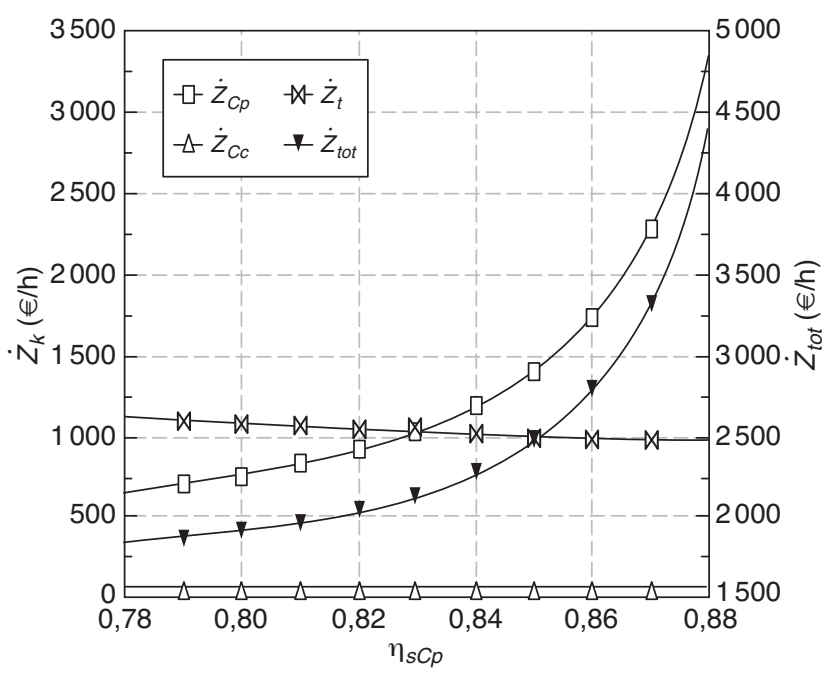

Figure 6

Influence du rendement isentropique du compresseur sur le coût d'amortissement du capital $\left(p_{2} / p_{1}=11,67\right.$; $\left.T_{3}=1460 \mathrm{~K} ; \eta_{s t}=0,874\right)$.

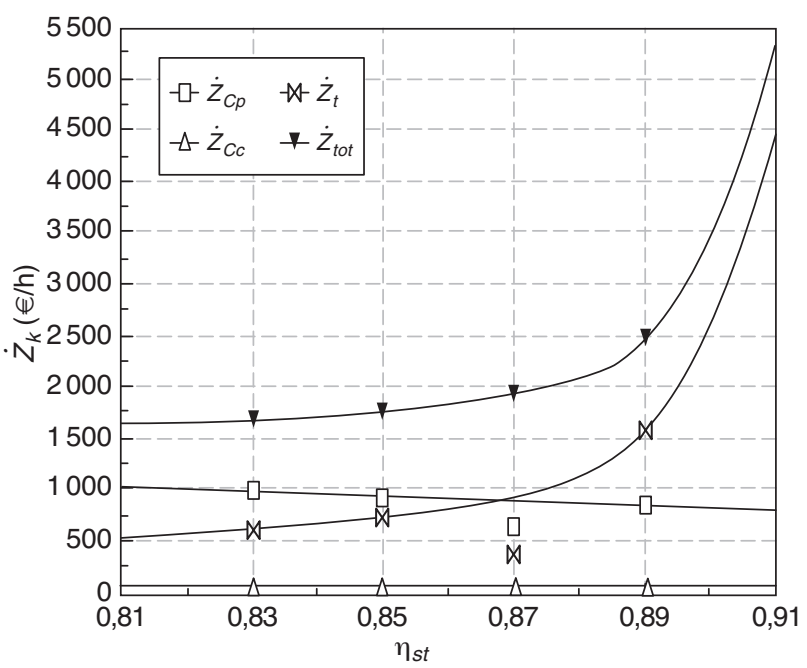

Figure 8

Influence du rendement isentropique de la turbine sur le coût d'amortissement du capital $\left(p_{2} / p_{1}=11,67 ; T_{3}=1460 \mathrm{~K}\right.$; $\left.\eta_{s C p}=0,815\right)$.

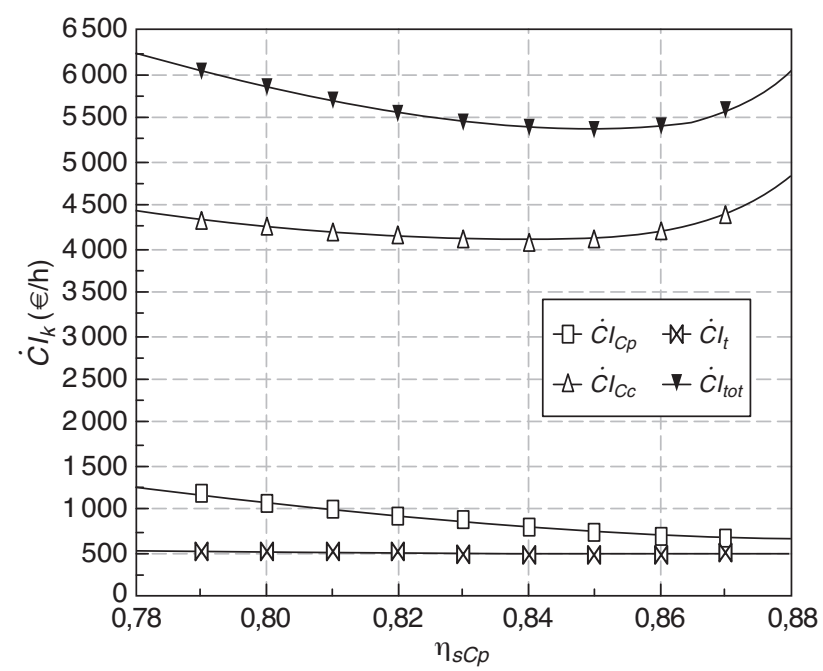

Figure 7

Influence du rendement isentropique du compresseur sur le coût de la destruction d'exergie $\left(p_{2} / p_{1}=11,67\right.$; $\left.T_{3}=1460 \mathrm{~K} ; \eta_{s t}=0,874\right)$.

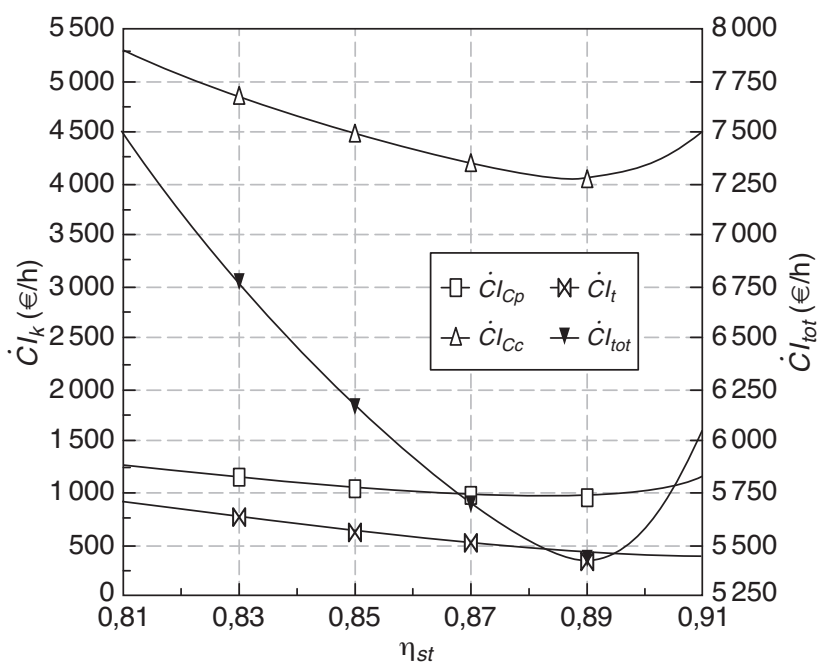

Figure 9

Influence du rendement isentropique de la turbine sur le coût de la destruction d'exergie $\left(p_{2} / p_{1}=11,67 ; T_{3}=1460 \mathrm{~K}\right.$; $\left.\eta_{s C p}=0,815\right)$.
- une diminution rapide du coût de la destruction d'exergie dans la chambre de combustion avec l'élévation de température $T_{3}$ (fig. 5);

- une réduction du coût de la destruction d'exergie de la chambre de combustion plus élevée que l'augmentation du coût d'amortissement du prix d'investissement de la turbine par élévation des rendements isentropiques (fig. 7-9).
Des résultats obtenus par cette étude exergo-économique sont présentés dans le tableau 1. Leur analyse permet d'affirmer que la zone avec le coût de destruction d'exergie le plus élevé est de loin la chambre de combustion. Cette zone se caractérise aussi par un facteur exergo-économique $f_{C c}$ extrêmement réduit, ce qui signifie que le coût d'amortissement du capital investi dans la chambre de combustion est très faible par rapport au coût de la destruction d'exergie due aux 
irréversibilités, d'où l'intérêt de la réduction du coût de la destruction d'exergie. La réduction des irréversibilités internes des processus dans la chambre de combustion peut être possible par l'élévation de la température moyenne de cette zone, c'est-à-dire par l'élévation des températures $T_{2}$ et $T_{3}$.

L'élévation de la température $T_{2}$ peut être réalisée dans cette configuration, seulement par l'augmentation du rapport de compression $p_{2} / p_{1}$ ce qui conduirait à l'augmentation rapide du coût du compresseur. Les données exergo-économiques du compresseur mettent en évidence un coût d'amortissement du capital investi $\dot{Z}_{C p}$ élevé, combiné avec une valeur élevée du facteur exergo-économique $f_{C P}$. Ces informations suggèrent d'accepter une élévation d'irréversibilités au compresseur qui conduirait à une réduction significative de l'investissement du capital. On propose dans ce sens la diminution de la performance du compresseur par la diminution de son rendement isentropique $\eta_{s C p}$ et du rapport de compression $p_{2} / p_{1}$.

En ce qui concerne la turbine, la valeur élevée du coût de la destruction d'exergie $\dot{C}_{t}$ suggère l'amélioration de la performance de ce composant, représentée par son rendement isentropique $\eta_{s t}$. Néanmoins, l'augmentation de son rendement $\eta_{s t}$ doit se faire avec précaution vue la pente de l'augmentation du coût de la turbine avec ce paramètre (fig. 8). Dans la procédure de recherche d'optimum du système global, l'analyse exergo-économique indique l'intérêt de l'élévation de la température des gaz brûlés en sortie de chambre de combustion $T_{3}$, de la diminution du rapport de compression $p_{2} / p_{1}$ et du rendement isentropique du compresseur et d'une faible augmentation du rendement isentropique de la turbine $\eta_{s t}$.

L'élévation de la température $T_{3}$ n'a que des conséquences positives sur le système. Dans le cas de la turbine, jusqu'à une certaine valeur de cette température, elle entraîne la diminution du coût d'investissement (fig. 4), due à l'augmentation du travail mécanique spécifique de la turbine et implicitement la diminution du débit de fluide véhiculé.

Les résultats de l'analyse exergo-économique correspondante à la première itération dans la procédure de recherche d'un optimum sont présentés dans le tableau 2. Si l'on compare les tableaux 1 et 2 on observe que la diminution du rapport de compression $p_{2} / p_{1}$ a un effet positif sur la diminution de la destruction d'exergie $\dot{I}_{C p}$. Cet effet est plus important que l'effet contraire dû à la réduction du rendement isentropique $\eta_{s C p}$. L'élévation de la température $T_{3}$ a eu l'effet attendu, conduisant à la diminution rapide de la destruction d'exergie dans la chambre de combustion, dont le coût reste cependant le plus élevé du système. Le facteur exergo-économique $f_{C c}$ est encore très faible ce qui impose aussi une augmentation de la température $T_{3}$. Le coût de l'amortissement du compresseur $\dot{Z}_{C p}$ et le facteur exergo-économique $f_{C p}$ correspondants à cette zone restent les plus élevés du système ce qui suggère une réduction du coût d'investissement par la diminution du

\section{TABLEAU 1}

Grandeurs exergo-économiques de la turbine à gaz pour $p_{2} / p_{1}=15 ; T_{3}=1200 \mathrm{~K} ; \eta_{s C p}=0,85 ; \eta_{s t}=0,85$

\begin{tabular}{c|c|c|c|c|c|c|c|c}
\hline Zone & $\begin{array}{c}\eta_{e x} \\
(\%)\end{array}$ & $\begin{array}{c}\dot{I} \\
(\mathrm{MW})\end{array}$ & $\begin{array}{c}y_{I} \\
(\%)\end{array}$ & $\begin{array}{c}\dot{Z} \\
(€ / \mathrm{h})\end{array}$ & $\begin{array}{c}\dot{C} I \\
(€ / \mathrm{h})\end{array}$ & $\begin{array}{c}\dot{C} I+\dot{Z} \\
(€ / \mathrm{h})\end{array}$ & $\begin{array}{c}r \\
(\%)\end{array}$ & $f$ \\
\hline$C c$ & 76,87 & 107,942 & 34,37 & 70,97 & 10529 & 10600 & 30,29 & 0,0067 \\
\hline$C p$ & 91,94 & 11,389 & 4,263 & 3970 & 2714 & 6684 & 21,6 & 0,594 \\
\hline$t$ & 92,59 & 20,268 & 6,454 & 1244 & 2576 & 3820 & 59,51 & 0,3256 \\
\hline
\end{tabular}

Le coût du produit du système global est $\dot{C}=3,366 € /$ s.

\section{TABLEAU 2}

$1^{\text {re }}$ itération : grandeurs exergo-économiques du système pour $p_{2} / p_{1}=13 ; T_{3}=1300 \mathrm{~K} ; \eta_{s C p}=0,83 ; \eta_{s t}=0,86$

\begin{tabular}{c|c|c|c|c|c|c|c|c}
\hline Zone & $\begin{array}{c}\eta_{e x} \\
(\%)\end{array}$ & $\begin{array}{c}\dot{I} \\
(\mathrm{MW})\end{array}$ & $\begin{array}{c}y_{I} \\
(\%)\end{array}$ & $\begin{array}{c}\dot{Z} \\
(€ / \mathrm{h})\end{array}$ & $\begin{array}{c}\dot{C} I \\
(€ / \mathrm{h})\end{array}$ & $\begin{array}{c}\dot{C} I+\dot{Z} \\
(€ / \mathrm{h})\end{array}$ & $\begin{array}{c}r \\
(\%)\end{array}$ & $f$ \\
\hline$C c$ & 77,66 & 92,685 & 31,86 & 54,85 & 6360 & 6415 & 30,72 & 0,00855 \\
\hline$C p$ & 90,71 & 10,865 & 3,735 & 1728 & 1691 & 3419 & 20,7 & 0,5054 \\
\hline$t$ & 93,8 & 13,367 & 4,595 & 1021 & 1199 & 2220 & 73,48 & 0,46 \\
\hline
\end{tabular}

Le coût du produit du système global est $\dot{C}=2,816 € /$ s.

TABLEAU 3

$2^{\mathrm{e}}$ itération : grandeurs exergo-économiques de la turbine à gaz pour $p_{2} / p_{1}=12 ; T_{3}=1400 \mathrm{~K} ; \eta_{s C p}=0,82 ; \eta_{s t}=0,87$

\begin{tabular}{c|c|c|c|c|c|c|c|c}
\hline Zone & $\begin{array}{c}\eta_{e x} \\
(\%)\end{array}$ & $\begin{array}{c}\dot{I} \\
(\mathrm{MW})\end{array}$ & $\begin{array}{c}y_{I} \\
(\%)\end{array}$ & $\begin{array}{c}\dot{Z} \\
(€ / \mathrm{h})\end{array}$ & $\begin{array}{c}\dot{C} I \\
(€ / \mathrm{h})\end{array}$ & $\begin{array}{c}\dot{C} I+\dot{Z} \\
(€ / \mathrm{h})\end{array}$ & $\begin{array}{c}r \\
(\%)\end{array}$ & $f$ \\
\hline$C c$ & 77,04 & 81,948 & 29,72 & 54,03 & 4709 & 4763 & 30,15 & 0,01134 \\
\hline$C p$ & 90,05 & 8,967 & 3,253 & 1075 & 1244 & 2319 & 20,6 & 0,4634 \\
\hline$t$ & 94,74 & 9,678 & 3,51 & 962,4 & 723,9 & 1686 & 85,53 & 0,57 \\
\hline
\end{tabular}

Le coût du produit du système global est $\dot{C}=2,512 € / \mathrm{s}$.

\section{TABLEAU 4}

$3^{\mathrm{e}}$ itération : grandeurs exergo-économiques de la turbine à gaz pour $p_{2} / p_{1}=11,5 ; T_{3}=1450 \mathrm{~K} ; \eta_{s C p}=0,81 ; \eta_{s t}=0,88$

\begin{tabular}{c|c|c|c|c|c|c|c|c}
\hline Zone & $\begin{array}{c}\eta_{e x} \\
(\%)\end{array}$ & $\begin{array}{c}\dot{I} \\
(\mathrm{MW})\end{array}$ & $\begin{array}{c}y_{I} \\
(\%)\end{array}$ & $\begin{array}{c}\dot{Z} \\
(€ / \mathrm{h})\end{array}$ & $\begin{array}{c}\dot{C} I \\
(€ / \mathrm{h})\end{array}$ & $\begin{array}{c}\dot{C} I+\dot{Z} \\
(€ / \mathrm{h})\end{array}$ & $\begin{array}{c}r \\
(\%)\end{array}$ & $f$ \\
\hline$C c$ & 77,32 & 78,418 & 28,75 & 66,2 & 4245 & 4311 & 29,79 & 0,01536 \\
\hline$C p$ & 89,48 & 8,584 & 3,147 & 823,9 & 1162 & 1986 & 20,1 & 0,415 \\
\hline$t$ & 95,16 & 8,436 & 3,093 & 1051 & 592,7 & 1643,7 & 92,6 & 0,639 \\
\hline
\end{tabular}

Le coût du produit du système global est $\dot{C}=2,454 € / \mathrm{s}$.

rapport de compression $p_{2} / p_{1}$ et du rendement isentropique $\eta_{s C p}$. L'augmentation du rendement isentropique de la turbine $\eta_{s t}$ a conduit à la réduction du coût total de cette zone $(\dot{C} I+\dot{Z})_{t}$ ce qui nous invite à une nouvelle augmentation de ce rendement. 
Pour la deuxième itération de la procédure de recherche d'optimum nous avons choisi les paramètres suivants :

$$
p_{2} / p_{1}=12 ; T_{3}=1400 \mathrm{~K} ; \eta_{s C p}=0,82 ; \eta_{s t}=0,87 \text {. }
$$

Les nouveaux résultats, obtenus par une seconde itération, sont présentés dans le tableau 3.

Après seulement trois itérations, on s'approche de la solution optimale. Les résultats correspondants sont présentés dans le tableau 4.

En prolongeant l'étude paramétrique pour la recherche de solution optimale dans le sens indiqué par les grandeurs exergo-économiques, c'est-à-dire la diminution du rapport de compression $p_{2} / p_{1}$ et du rendement isentropique du compresseur $\eta_{s C p}$ et l'élévation de la température $T_{3}$ et du rendement isentropique de la turbine $\eta_{s t}$, le coût du produit global commence à croître, ce qui marque l'éloignement de l'optimum de fonctionnement.

On observe que le résultat obtenu à l'aide de l'analyse exergo-économique est proche de celui obtenu par la "Direct search method". Par contre, contrairement à la méthode mathématique qui cherche un point extrême de la fonction objectif et qui se comporte comme une "boîte noire", la méthode exergo-économique nous permet de suivre le modèle physique, indiquant les pas à suivre pour trouver une solution optimale.

La procédure d'optimisation exergo-économique se base sur une stratégie qui permet la connaissance des processus physiques et économiques qui ont lieu dans le système. Leur maitrise permettra de réduire la dépense totale du système.

Dans le cas présenté ci-dessus (Tab.4) on observe que les valeurs de la destruction d'exergie dans la chambre de combustion $\left(\dot{I}_{C c}=78,418 \mathrm{MW}\right)$ et du coefficient de destruction d'exergie par la dépense de combustible $\left(y_{I}=28,75 \%\right)$ sont encore très élevées.

Ayant remarqué que la destruction d'exergie dans la chambre de combustion diminue avec l'élévation de la température moyenne thermodynamique des processus, il est évident qu'on va essayer de réduire le coût par l'élévation de cette température.

L'élévation, par la suite, de la température $T_{3}$, au delà d'une certaine valeur, a comme effet une augmentation rapide du prix de la turbine (fig. 5), ce qui nous éloigne de la solution optimale.

Afin de réduire encore les irréversibilités dans la chambre de combustion il faudrait majorer la température $T_{2}$, non pas par l'élévation du rapport de compression $p_{2} / p_{1}$ qui pourrait entraîner un coût du compresseur plus élevé et implicitement un coût de destruction d'exergie dans la chambre de combustion plus élevé par l'élévation du coût unitaire du combustible de cette zone $\left(c_{C b, C c}\right)$.

L'analyse exergo-économique indique une perte d'exergie importante avec l'évacuation des gaz brûlés en sortie de turbine, qui atteint une valeur de $\dot{E} x_{4}^{T O T}=120 \mathrm{MW}$ (fig. $1 \mathrm{~b}$ ), pour la solution optimale.

Étant donnée que la perte d'exergie thermique avec les gaz brûlés est très importante, il est judicieux d'augmenter la température $T_{2}$ de l'air en sortie de compresseur par la récupération d'une partie de cette exergie perdue.

\section{TURBINE À GAZ AVEC PRÉCHAUFFAGE D’AIR}

Une solution technologique utilisée aujourd'hui pour l'amélioration du rendement de la turbine est l'utilisation d'un échangeur récupérateur qui servira au préchauffage de l'air comprimé à l'entrée de la chambre de combustion (fig. 10).

En termes exergétiques, cela se traduit par une récupération d'exergie thermomécanique des gaz évacués par la turbine. Les effets positifs sont la réduction de la destruction d'exergie de la chambre de combustion, la réduction de la perte d'exergie et, par conséquent, l'augmentation du rendement exergétique global.

Le point de départ de la méthode de recherche d'optimum est donné par les variables de décision optimales obtenues pour la turbine à gaz simple. Le rendement du préchauffeur est considéré égal à $\eta_{P a}=0,8$.

Les paramètres exergo-économiques du modèle sont présentés dans le tableau 5 . Leur analyse permet de remarquer que le coût le plus élevé $\dot{C} I+\dot{Z}$ est au niveau de la chambre de combustion et le plus bas est au niveau du compresseur.

Le facteur exergétique $(f)$ très faible dans la chambre de combustion montre que son coût est dû exclusivement à la destruction d'exergie, indiquant la nécessité d'élever $T_{2}$ par préchauffage, donc par augmentation du rendement de préchauffage $\eta_{P a}$. La nécessité d'augmenter le rendement de

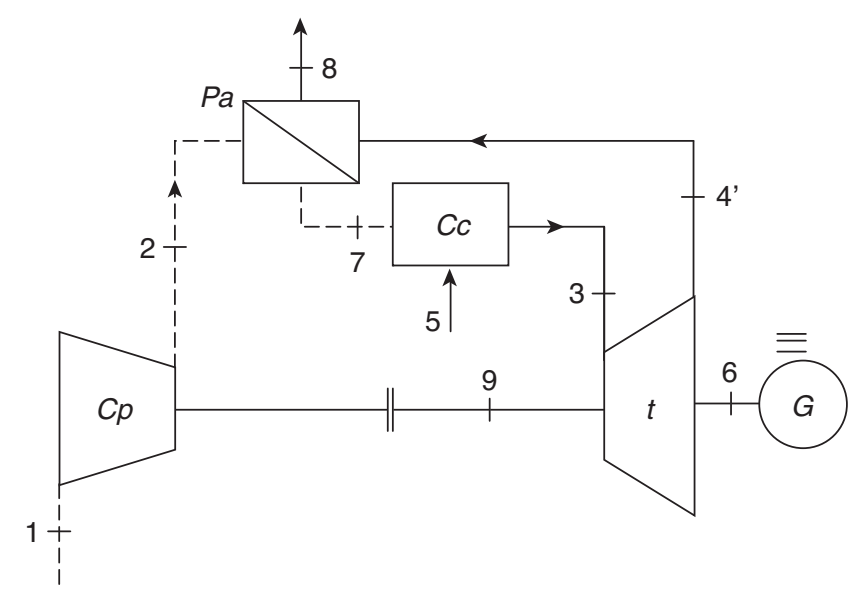

Figure 10

Turbine à gaz à récupération. 
préchauffage est indiquée par le niveau très élevé du coefficient d'élévation relative de son coût $r_{P a}$ ainsi que par son rendement exergétique faible.

\section{TABLEAU 5}

Grandeurs exergo-économiques de la turbine à gaz pour $p_{2} / p_{1}=11,5 ; T_{3}=1450 \mathrm{~K} ; \eta_{s C p}=0,81 ; \eta_{s t}=0,88 ; \eta_{P a}=0,8$

\begin{tabular}{c|c|c|c|c|c|c|c|c}
\hline Zone & $\begin{array}{c}\eta_{e x} \\
(\%)\end{array}$ & $\begin{array}{c}\dot{I} \\
(\mathrm{MW})\end{array}$ & $\begin{array}{c}y_{I} \\
(\%)\end{array}$ & $\begin{array}{c}\dot{Z} \\
(€ / \mathrm{h})\end{array}$ & $\begin{array}{c}\dot{C} I \\
(€ / \mathrm{h})\end{array}$ & $\begin{array}{c}\dot{C} I+\dot{Z} \\
(€ / \mathrm{h})\end{array}$ & $\begin{array}{c}r \\
(\%)\end{array}$ & $f$ \\
\hline$C c$ & 81,42 & 62,01 & 28,4 & 36,24 & 5308 & 5344,24 & 22,98 & 0,00678 \\
\hline$C p$ & 89,48 & 9,42 & 4,315 & 904,4 & 1119 & 2023,4 & 21,26 & 0,4469 \\
\hline$t$ & 95,43 & 8,475 & 3,88 & 1235 & 892,2 & 2127,2 & 12,84 & 0,5806 \\
\hline$P a$ & 32,26 & 4,343 & 1,99 & 58,29 & 457,2 & 515,5 & 90,5 & 0,1131 \\
\hline
\end{tabular}

Le coût du produit du système global est $\dot{C}=2,15 € / \mathrm{s}$.

La diminution de la destruction d'exergie dans la chambre de combustion sera possible par l'élévation de la température $T_{3}$, dont la valeur est en même temps limitée par le coût élevé de la turbine.

Les coûts d'investissement de la turbine et du compresseur seront diminués par la réduction du rapport $p_{2} / p_{1}$. La diminution du coût associé à la turbine pourrait être possible par la diminution de son rendement isentropique alors que la destruction d'exergie du compresseur peut être diminuée par l'augmentation de son rendement isentropique $\eta_{s C p}$.

La procédure d'optimisation exergo-économique de la nouvelle installation conduit à une solution optimale définie par les paramètres suivants :

$p_{2} / p_{1}=3,64 ; T_{3}=1470 \mathrm{~K} ; \eta_{s C p}=0,84 ; \eta_{s t}=0,85 ; \eta_{P a}=0,98$.

Les résultats de l'analyse exergo-économique qui représente la solution optimale sont présentés dans le tableau 6 . On obtient un coût du produit de ce système bien inférieur à celui de la turbine à gaz simple $\dot{C}=1,56 € / \mathrm{s}$.

\section{TABLEAU 6}

Grandeurs exergo-économiques de la turbine à gaz avec échangeur récupérateur de préchauffage de l'air pour

$p_{2} / p_{1}=3,64 ; T_{3}=1470 \mathrm{~K} ; \eta_{s c}=0,84 ; \eta_{s t}=0,85 ; \eta_{P a}=0,98$

\begin{tabular}{c|c|c|c|c|c|c|c|c}
\hline Zone & $\begin{array}{c}\eta_{e x} \\
(\%)\end{array}$ & $\begin{array}{c}\dot{I} \\
(\mathrm{MW})\end{array}$ & $\begin{array}{c}y_{I} \\
(\%)\end{array}$ & $\begin{array}{c}\dot{Z} \\
(€ / \mathrm{h})\end{array}$ & $\begin{array}{c}\dot{C} I \\
(€ / \mathrm{h})\end{array}$ & $\begin{array}{c}\dot{C} I+\dot{Z} \\
(€ / \mathrm{h})\end{array}$ & $\begin{array}{c}r \\
(\%)\end{array}$ & $f$ \\
\hline $\mathrm{Cc}$ & 86,44 & 55,5 & 32,17 & 64,67 & 3695 & 3760 & 15,96 & 0,1273 \\
\hline$C p$ & 86,1 & 8,43 & 3,9 & 331,9 & 580,73 & 912,6 & 23,51 & 0,3637 \\
\hline$t$ & 95,43 & 7,69 & 3,335 & 566,3 & 442,2 & 1010 & 11,76 & 0,5604 \\
\hline$P a$ & 83,62 & 8,09 & 4,69 & 311,3 & 624,8 & 936 & 21,77 & 0,3325 \\
\hline
\end{tabular}

Dans ce cas, la destruction d'exergie dans la chambre de combustion a baissé de $29 \%$ et le coût de cette destruction a baissé de $13 \%$, ce qui a provoqué une diminution du coût du produit final de $37 \%$. Ainsi le rendement exergétique global a augmenté de 36,4\% par rapport à la turbine à gaz simple.

\section{CONCLUSIONS}

L'objectif de cet article est de souligner l'intérêt de l'analyse exergétique et de l'optimisation exergo-économique qui oriente le choix de composants et de paramètres de fonctionnement d'un système énergétique pour minimiser le coût global.

Le potentiel intéressant de cette approche a été illustré pour une turbine à gaz simple et à récupération pour laquel on dispose de nombreuses corrélations exergo-économiques définissant le coût d'investissement de ses composants.

L'optimisation exergo-économique offre en plus de l'optimisation thermo-économique une stratégie cohérente de recherche d'optimum, qui conduit à la maîtrise des procédés énergétiques internes des différents composants du système étudié.

\section{RÉFÉRENCES}

1 Lewis R.M., Torczon V., Trosset M.W. (2000) Direct search methods; then and now, J. Comput. Appl. Math. 124, 1-2, 191-207.

2 Davidon W.C. (1991) Variable metric method for minimization, Society for Industrial and Applied Mathematics, SIAM J. Optim. 1, 1, 1-17.

3 Frangopoulos C.A. (1983) Thermoeconomic Functional Analysis: A Method for the Optimal Design or Improvement of Complex Thermal Systems, PhD Thesis, Georgia Institute of Technology, Atlanta, GA.

4 El-Sayed Y.M. (1988) Approaches to the Design and Optimization of Thermal Systems, AES-7, Wepfer W.J. and Moran M.J. (eds), ASME, New York.

5 Bejan A., Tsatsaronis G., Moran M. (1996) Thermal Design and Optimization, John Wiley.

6 Grosu L. (2000) Contribution à l'optimisation thermodynamique et économique des machines à cycle inverse à deux et trois réservoirs de chaleur, PhD Thesis, Institut National Polytechnique de Lorraine, Nancy.

7 Dobrovicescu A., Feidt M., Grosu L., Mc Govern J. (2006) Analyse exergétique d'un système de turbine à gaz, Bull. Scientifique de l'Univ. Politehnica de Timişoara, Série Génie Mécanique, Tome 51 (65), 1, 13-23.

8 El-Sayed Y.M., Evans R.B. (1970) Thermoeconomics and the Design of Heat Systems, J. Eng. Power, January, 27-35.

9 Reistad G.M., Gaggioli R.A. (1978) Available Energy Costing, American Chemical Society, Miami, Fl., Sept.

10 Borel L. (1979) Théorie générale de l'exergie et applications pratiques, $1^{\text {re }}$ partie : Bilan exergétique, coénergie et coenthalpie, Entropie, Nr. 85, Jan.

11 Lozano M.A. (1992) Analisis exergetico, Maquinas y Motores Termicos, Dpto de Ingenieria Mecanica, Universidad de Zaragoza.

12 Valero A., Lozano M.A. (1997) An Introduction to Thermoeconomics, in Developments in the Design of Energy Systems, Boeh R.F. (ed.), Cambridge University Press, Cambridge, Mass, pp. 203-233. 
13 Verda. V., Borchiellini R. (2004) Exergetic and economic evaluation of control strategies for a gas turbine plant, Energy 29, 2253-2271.

14 Ertesvag I.S., Kvarnsdal H.M., Bolland O. (2005) Exergy analysis of a gas-turbine combined-cycle power plant with precombustion $\mathrm{CO}_{2}$ capture, Energy 30, 5-39.

15 Ensinas A.V., Nebra S.A., Lozano M.A., Sierra L. (2006) Optimization of thermal energy consumption in sugar cane factories, ECOS 2006, Proceedings of the 19th International Conference on Efficiency, Cost, Optimization, Simulation and Environmental Impact of Energy Systems, Crete, Greece, 12-14 July, pp. 569-576.

16 Dobrovicescu A., Tsatsaronis G. (2006) Exergy destruction due to friction in heat exchangers - A refrigeration system case study, ECOS 2006, Proceedings of the 19th ASME International Conference on Efficiency, Cost, Optimization, Simulation and Environmental Impact of Energy Systems, pp. 165-173.

17 Dobrovicescu A. (2005) Exergetic analysis of a cryogenic refrigeration system, The ASME 18th International Conference on Efficiency, Cost, Optimization, Simulation and Environmental Impact of Energy Systems Shaping our Future Energy Systems ECOS 2005, Trondheim, Norway, June 20-23, pp. 345- 352.

18 Feidt M. et al. (2006) Comparaison entre le cycle simple de Brayton avec apport thermique imposé et avec contrainte de température maximale, Oil Gas Sci.Technol. 61, 2, 237-247.
19 Dobrovicescu A. et al. (2011) The decomposition method in the exergoeconomic optimization of energy systems, Revue Roumaine de Sciences Techniques Série Électrotechnique et Énergétique 4, 428-437, Publisher: Romanian Academy, Publishing House of the Romanian Academy.

20 Tsatsaronis G., Lin L., Pisa J. (1993) Exergy Costing in Exergoeconomics, J. Energy Resour. Technol. 115, 9-16.

21 Lozano M.A., Valero A. (1993) Theory of the exergetic cost, Energy 18, 9, 939-960.

22 Chejne F., Restrepo J.A. (2003) New rules for the exergoeconomic optimization methodology, Energy 28, 993-1003.

23 Dobrovicescu A. (2007) Principles of exergoeconomic analysis, Politehnica Press, Bucaharest.

24 Tsatsaronis G., Moran M.J. (1997) Exergy-Aided Cost Minimization, Energy Convers. Manage. 38, 15-17, 1535-1542.

Manuscrit final reçu en février 2012 Publié en ligne en septembre 2012 